\title{
Joint iterative channel estimation and decoding under pulsed radio frequency interference condition
}

\author{
Patcharin INSOM*, Rongke LIU*, Ruifeng DUAN*, Yi HOU* and Pisit BOONSRIMUANG ${ }^{\dagger}$ \\ ${ }^{*}$ School of Electronic and Information Engineering \\ Beihang University,Beijing, China 100191 \\ Email: insom.patcharin@gmail.com \\ ${ }^{\dagger}$ Telecommunication Engineering Department, Faculty of Engineering \\ King Mongkut's Institute of Technology Ladkrabang, Bangkok, Thailand 10520
}

\begin{abstract}
Pulsed Radio Frequency Interference (RFI) has severely effected on communication system in common. Even though Low-Density-Parity-Check (LDPC) code which has the decoding performance to approach the Shannon Limit and it is designed as a powerful forward-error-correction (FEC) code in the Additive White Gaussian Noise (AWGN) channel, simulation results show that the performance of LDPC decoder is degraded when exposed to the pulsed RFI. According to such a pulsed RFI impact, joint iterative channel estimation and decoding technique is proposed in this paper so as to decrease the effect of pulsed RFI while less complicated in processing. The proposed method decreases the complexity by implementing the simple way of SNR estimation and improves the efficiency and applying joint iterative technique between channel estimation and decoding. For optimal decoder performance, Signal-to-Noise Ratio (SNR) estimation must be as accurate as possible. Because computed in each time of iterative decoder, noise variance has been always change resulting in the performance of LDPC decoder has better in efficiency. The simulation results show that both less complexity and higher outcomes can be achieved by this proposed approach.
\end{abstract}

Keywords-LDPC decoding, Pulsed RFI, Pulsed RFI impact, Joint iterative, Channel estimation.

\section{INTRODUCTION}

Reliability is an important thing in communication systems. Undesirable signal should be eliminated. Not only planetary communications systems, but also satellite communications systems are commonly interrupted by pulsed RFI. Several researches which investigate the impact of pulsed RFI on LDPC decoder performance found that regardless of the level of SNR, the Bit-Error-Rate (BER) is always large [1]. Pulsed RFI frequently has the duty cycles in the order of $5 \%$ or smaller. This significantly decreases the impact on receiver performance compared to continuous interference with the same power and centre frequency [6]. In addition, pulsed RFI randomly turns up in the form of block and the noise variance varies with time. Currently, several applications, such as ISS ACS Transponders, 4th Gen Transponders, Integrated Receivers and satellite communication receiver, are involved with pulsed RFI, resulting in wide awareness of the impact of pulsed RFI.

LDPC codes, invented by Gallager, achieve near capacity performance in a wide class of channels [5]. Generally, various simulations and applications are based on AWGN channel.
However, our simulation is unable to consider being only AWGN channel because of pulsed RFI appearing. As mentioned earlier, the noise variance of pulsed RFI is time varying so, SNR evaluation must perform on fly.

Several researchers have investigated a number of SNR estimation algorithms [2]. Although all of them work very well when the SNR is high, their performance suffers at negative SNR. The iterative SNR estimation [3] is rest on an iterative solution for the maximum likelihood estimation of the amplitude from which the SNR is competent to compute. Its results show that it exhibits a lower bias and normalized MSE than other techniques and that the useful range extends to negative SNR. However, the iterative SNR estimation also has flaw because of demanding a lot of complicated processes and taking a large of calculating time depending on the number of iteration. In the co-channel interference scenario and nonAWGN channel, an initial Log-Likelihood-Ratio (LLR) has been developed in many ways [10-14]. This work constructs the initial LLR based on probability density function (pdf.) [4]. The different initial LLR for LDPC codes using the density evolution technique to analyze. Nowadays, joint iterative technique have been extensively implement in several applications [15][16]. The proposed method named joint iterative channel evaluation and decoding technique which has been developed to re-processing SNR estimation during each time of LDPC decoder processes in other words we combine working process between SNR estimation and LDPC decoder.

The rest of this paper is organized as follows. Section II presents the system model, the pulsed RFI model and the influence of pulsed RFI on LDPC decoder performance. Section III, which reviews SNR estimation techniques, consists of second order moment SNR estimation and the iterative SNR estimation. In section IV, the joint iterative channel estimation and decoding approach is presented. The simulation results of the proposed technique which demonstrates in section $\mathrm{V}$ are compared to conventional and another complex decoding technique in term of BER. The last section is conclusion.

\section{SYSTEM MODEL AND PULSED RFI MODEL}

The whole system illustrates as Figure 1. and (1) where $x_{k}$ is transmitted sequences and $y_{k}$ is received sequences. The transmitted signals $\left(x_{k}\right)$ are interrupted by pulsed RFI $\left(n_{r f i, k}\right)$ and AWGN $\left(n_{\text {awgn, }}\right)$ which both of them have zero mean, 
but possess difference in noise variance. Distribution of AWGN and pulsed RFI are capable of representing as $N\left(0, \sigma_{1}^{2}=\sigma^{2}+\right.$ $\Delta \sigma)$ and $N\left(0, \sigma_{2}^{2}\right)$ respectively. Practically, because most of the channel is time varying SNR mismatch over an AWGN channel, the noise variance is explained as $\sigma_{1}^{2}=\sigma^{2}+\Delta \sigma$. When $\sigma^{2}$ signify as the noise variance of AWGN and $\Delta \sigma$ is symbolic of the random walk signal of AWGN.

$$
y_{k}=x_{k}+n_{\text {awgn }, k}+g(t) * n_{r f i, k}
$$

where

$n_{\text {awgn,k }} \sim N\left(0, \sigma_{1}^{2}=\sigma^{2}+\Delta \sigma\right)$

$n_{r f i, k} \sim N\left(0, \sigma_{2}^{2}\right)$ Figure 2. shows pulsed RFI in single

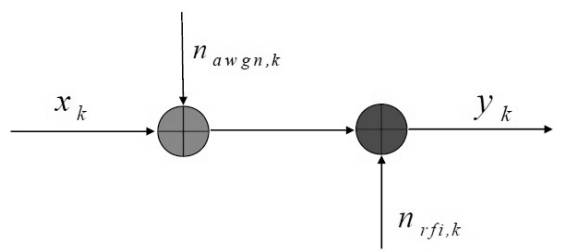

Figure 1. System model.

source. Generally, pulsed RFI randomly turns up throughout the length of $x_{k}$ and has duty cycles $3-5 \%$ of the code length. In addition, pulsed RFI has the noise variance changing with time. Pulse train function $(g(t))$ is assumed to be periodic

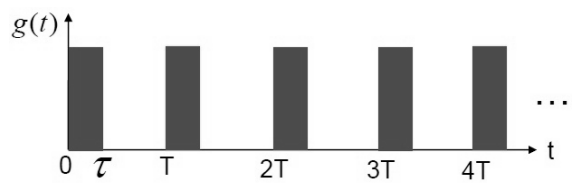

Figure 2. Pulsed RFI model.

gating function with pulse repetition rate, rate $1 / \mathrm{T}$ and duty cycle $\tau / \mathrm{T}$ [1]. Figure 3 . demonstrates the influence of pulsed RFI on LDPC decoder performance. It is found that whatever $\mathrm{SNR}$ is, BER under pulsed RFI environment is always high.

\section{Signal-TO-Noise Ratio (SNR) EStimation TECHNIQUES}

Various digital communication applications, such as power control, bit error estimation, and turbo decoding, involve the knowledge of the SNR. For optimal performance, SNR estimation must be as accurate as possible. Several techniques have been proposed for SNR estimation. In this work, two techniques which are taken into the simulation.

The second order moment SNR estimation [2] is popular in several applications because it provides a simple processing. SNR is capable of computing from the ratio of the signal mean $(A)$ squared and the noise variance $\left(\sigma^{2}\right)$ which both of them are expressed as

$$
\begin{gathered}
A=\frac{1}{N} \sum_{k=1}^{N}\left|y_{k}\right| \\
\sigma^{2}=\frac{1}{N} \sum_{k=1}^{N}\left(y_{k}-A\right)^{2}
\end{gathered}
$$

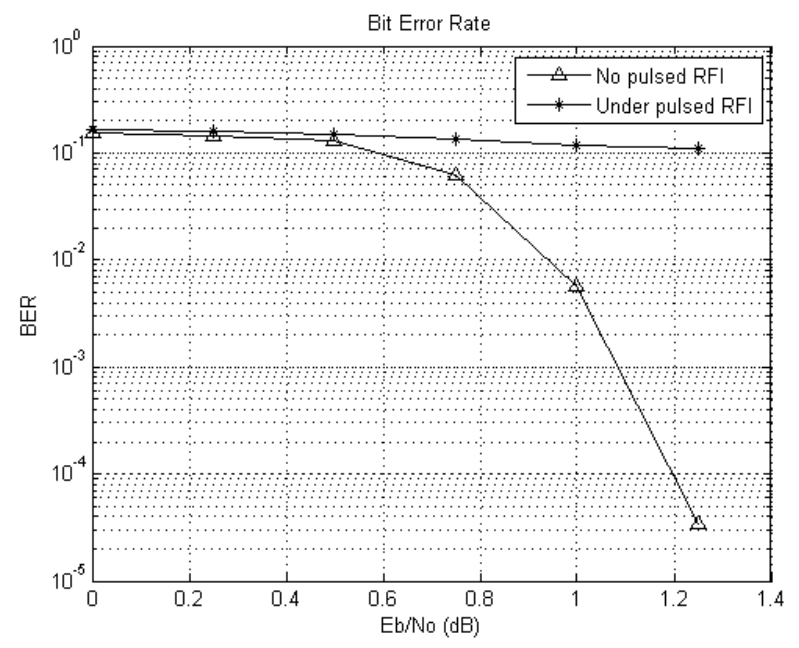

Figure 3. Pulsed RFI impact on LDPC decoder performance(the AR4JA (8192, 4096) code).

The iterative SNR estimation [3] is formed from the maximumlikelihood principle that has even lower estimation bias at low and negative SNR. They develop an iterative search algorithm to find the amplitude that satisfies (4) by defining function $F(x)$ as (5). They must perform the iterative algorithm to find the root of $F(x)$ where $F(x)=0$. the SNR is able to estimate as $\frac{x^{2}}{1-x^{2}}$.

$$
A=\frac{1}{N} \sum_{k=1}^{N} y_{k} \operatorname{th}\left(\frac{A y_{k}}{\sigma^{2}}\right)
$$

where $\operatorname{th}(x)=\left(e^{x}-e^{-x}\right) /\left(e^{x}+e^{-x}\right)$

$$
F(x)=x-\frac{1}{N} \sum_{k=1}^{N} y_{k} \operatorname{th}\left(\frac{x y_{k}}{\sigma^{2}}\right)
$$

Therefore, the complexity in computation of two methods have significant difference. In other words the second order moment SNR estimation technique is less computational processing than the iterative one.

\section{JOINT ITERATIVE CHANNEL ESTIMATION AND DECODING}

The system model which mentioned in the section II points out that both AWGN and pulsed RFI are capable of explaining as the normal distribution. Based on the pdf of the distribution, we are able to form the initial LLR of simulated channel. If the assumed noise pdf has distribution as

$$
f(n)=\frac{1-\epsilon}{\sqrt{2 \pi \sigma_{1}^{2}}} e^{-\frac{n^{2}}{2 \sigma_{1}^{2}}}+\frac{\epsilon}{\sqrt{2 \pi \sigma_{2}^{2}}} e^{-\frac{n^{2}}{2 \sigma_{2}^{2}}}
$$

where $\sigma_{2}^{2}>\sigma_{1}^{2}$

Contaminated Gaussian Log-Likelihood-Ratio (CGLLR) [4] has been constructed. CGLLR provides initial information more accurate than implementing Gaussian LLR. CGLLR is 
re-written as the follow

$$
L_{C G\left(y_{k}\right)}=\log \left(\frac{\frac{1-\epsilon}{\sqrt{2 \pi \sigma_{1}^{2}}} e^{-\frac{\left(y_{k}-1\right)^{2}}{2 \sigma_{1}^{2}}}+\frac{\epsilon}{\sqrt{2 \pi \sigma_{2}^{2}}} e^{-\frac{\left(y_{k}-1\right)^{2}}{2 \sigma_{2}^{2}}}}{\frac{1-\epsilon}{\sqrt{2 \pi \sigma_{1}^{2}}} e^{-\frac{\left(y_{k}+1\right)^{2}}{2 \sigma_{1}^{2}}}+\frac{\epsilon}{\sqrt{2 \pi \sigma_{2}^{2}}} e^{-\frac{\left(y_{k}+1\right)^{2}}{2 \sigma_{2}^{2}}}}\right)
$$

Where

- $\sigma_{1}^{2}=\sigma^{2}+\Delta \sigma$, is the noise variance of Gaussian part of the pdf.

- $\quad \sigma_{2}^{2}$ is the noise variance of the contaminating heavytailed pdf. Chosen ranges are $1.0 \leq \sigma_{2}^{2} \leq 4.0$.

- $\epsilon$ is the percentage of sample from the heavy-tailed pdf. $\epsilon$ can range from $0<\epsilon \leq 0.5$ with value greater than 0.3 occurring with very low probability.

To implement this kind of LLR equation, two parameters must be computed.

The first, the percentage of sample from the heavy-tailed pdf $(\epsilon)$ or pulsed RFI part. Algorithms that are based on higher order moments are used to detect the presence of pulsed RFI [7][8]. Figure 4. illustrates detection of pulsed RFI technique by second order moment. Let $\mathrm{N}$ is code length and $\mathrm{G}$ is

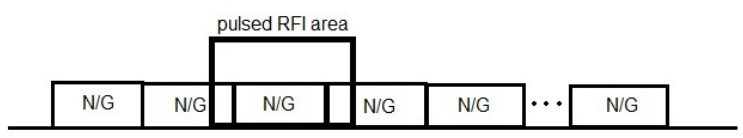

Figure 4. Detection pulsed RFI by the second order moment.

number of group. $\mathrm{N}$ is divided into $\mathrm{G}$ groups; so, each group is composed of N/G samples. Because the second order moment $\left(M_{2}\right)$ value of pulsed RFI area is higher than any other region, we take the advantage from this point to capture the area that pulsed RFI appearing. The second order moment $\left(M_{2}\right)$ of every group is calculated and the group which has the largest $M_{2}$ value is capable of considering to be pulsed RFI region. Hence, number of sample of pulsed RFI is approximately equal to N/G. To ensure that all pulsed RFI samples are detected, a few samples both left and right side of that group are included. In other words the pulsed RFI region is approximately equal to the area of group which it has the highest $M_{2}$ value including its side-band. Further precision may divide $\mathrm{N}$ into several groups and each group contains smaller samples. Applying the same technique; find $M_{2}$ of every group then pulsed RFI area may contain more than one group which have the highest $M_{2}$ value respectively. In practice, the second order moments $\left(M_{2}\right)$ are estimated by their respective time averages for both real and complex channels as

$$
M_{2} \approx \frac{1}{N} \sum_{k=1}^{N}\left|y_{k}\right|^{2}
$$

The second factor that needs to compute is the noise variance of Gaussian part of the pdf $\left(\sigma_{1}^{2}=\sigma^{2}+\Delta \sigma\right)$ because the channel status information is unknown. In various applications, the second order moment SNR estimation is used because it is the simple way to obtain $\sigma_{1}^{2}$ and does not take time to calculate a lot. However, the channel in this work is not same as other works since pulsed RFI also appears in the channel. In this environment, the second order moment SNR estimation is unable to provide very accurate value. The iterative SNR technique offers the noise variance value very near to the ideal value. Therefore, LDPC decoder performance has an exceptional efficiency. However, the iterative SNR estimation demands a large amount of processing time for the result depending on the number of iteration.

To obtain higher performance than conventional method which deployed the second order moment SNR estimation and less processing time than the complex method which implemented the iterative SNR estimation, the proposed method has been created.

Let consider in case of all BPSK modulated information in real channel is 1 or -1 , the probability density function of a received vector $\left(y_{1}, y_{2}, \ldots y_{N}\right)$ is expressed as

$$
f_{n}\left(y_{1}, y_{2}, \ldots, y_{N}\right)=\prod_{i=1}^{N} \frac{1}{\sqrt{2 \pi} \sigma} e^{-\frac{\left(y_{i}-A\right)^{2}}{2 \sigma^{2}}}
$$

where transmitted signal taking values from $\{A,-A\}$ and let,

$$
\frac{\partial f_{n}\left(y_{1}, y_{2}, \ldots, y_{N}\right)}{\partial A}=0
$$

Therefore,

$$
\frac{\partial \ln \left[f_{n}\left(y_{1}, y_{2}, \ldots, y_{N}\right)\right]}{\partial A}=0
$$

From (9) and (11), the noise variance is computed

$$
\sigma^{2}=\frac{1}{N} \sum_{i=1}^{N}\left(y_{i}-\frac{1}{N} \sum_{i=1}^{N} y_{i}\right)^{2}
$$

Giving concentration on (2),(3) and (12), it is found that both the signal mean value $(A)$ and the noise variance $\left(\sigma^{2}\right)$ of this case are as same as the second order moment SNR estimation. Therefore, the second order moment SNR estimation technique is the best solution to evaluate SNR of this kind modulated information.

Even though initial information are randomly generated, we are able to modify modulated information to be the new information which contains only 1 or -1 as same as the former case. The proposed approach is constructed from this principle. Joint iterative channel estimation and decoding are generated

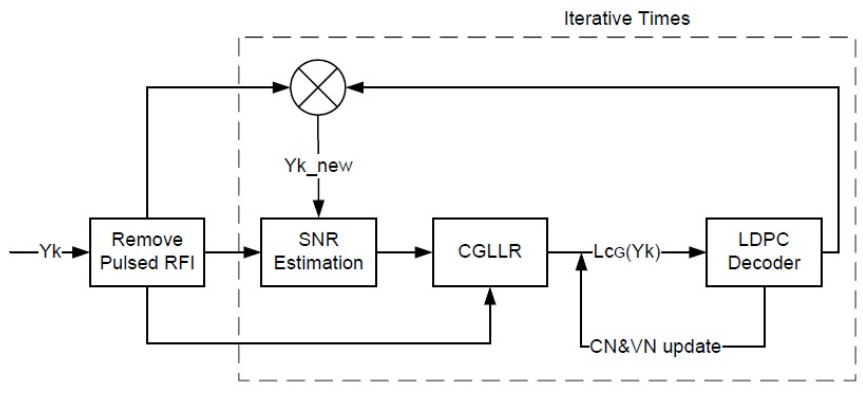

Figure 5. Schematic block diagram of the proposed technique. 
based on the fundamental that each iterative time of decoding process, the noise variance $\left(\sigma_{1}^{2}\right)$ is re-computed by the second order moment SNR estimation of the modified information. Schematic of the proposed technique shows as Figure 5.

Taking the advantage from the second order moment $\left(M_{2}\right)$ as mentioned earlier, the received signal $\left(y_{k}\right)$ is erased pulsed RFI area. The received signal after deleting pulsed RFI region denote as $y_{k}$ noRFI. The second step is the noise variance $\left(\sigma_{1}^{2}\right)$ evaluation. On the one hand, if it is the first time of LDPC decoder iterations, $y_{k \_n o R F I}$ will be used to perform the noise variance calculation. On the other hand, the noise variance is computed from $y_{k \_n e w}$. For optimal LLR, $\mathrm{L}_{C G}\left(y_{k}\right)$ is determined in (7). We carry out the LDPC decoder only once iterative time. The results consist of of hard-decision output, values of check node $(\mathrm{CN})$ and variable node $(\mathrm{VN})$ updating. Hard-decision outputs are feed back to multiply with the preeliminated pulsed RFI signal $\left(y_{k}\right)$ resulting in the new received signal $\left(y_{k \_n e w}\right)$. In order to keep LDPC decoder processes, values of check nodes $(\mathrm{CN})$ and variable nodes (VN) updating are also feed back so as to use in the initial state of the next LDPC iteration. In the check stopping criteria step, if the number of iterative time is not equal to the maximum LDPC iterations or decoding error is not equal to zero, go back to step 2.) and increases the number of iterations by one until reach the stopping criterion.

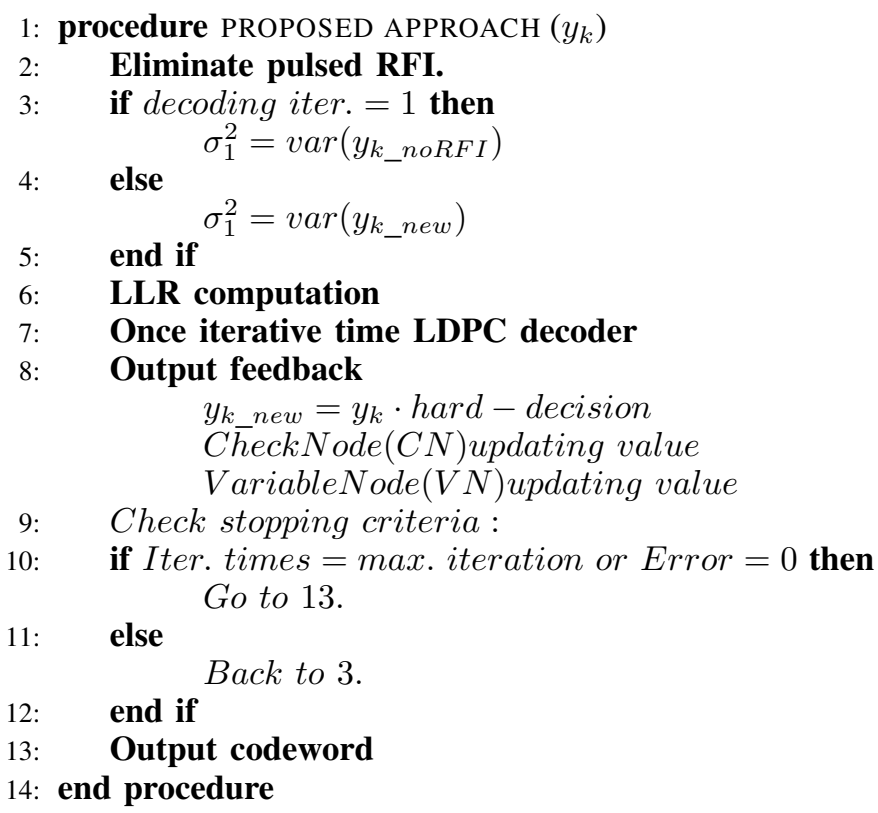

\section{SimUlation RESULTS AND DISCUSSIONS}

The LDPC code studied here is the AR4JA $(8192,4096)$ code recommended by the Consultative Committee for Space Data Systems (CCSDS) and it has been chosen for some deep space applications [9]. In the simulation, we set the noise variance of the contaminating heavy-tailed pdf $\left(\sigma_{2}^{2}\right)$ by 4.0 and set the random walk signal of AWGN $(\Delta \sigma)$ by $10 \%$ of the noise variance of AWGN part $\left(\sigma_{1}^{2}\right)$.

In this section, BER of the joint iterative channel estimation and decoding or the proposed approach is presented in order to compare with two methods. Conventional technique performs noise variance of AWGN part $\left(\sigma_{1}^{2}\right)$ estimation by second order moment SNR estimation based on hard-decisions of the previous codeword which they are feed back to multiply with that codeword to eliminate modulated information. Complex technique implements iterative SNR estimation which it give a high evaluation performance to estimate $\sigma_{1}^{2}$.

Figure 6. depicts that the proposed technique has only about $0.2 \mathrm{~dB}$ at BER $=10^{-3}$ different from the SNR know and complex method. The efficiency of the proposed approach is close to the performance of ideal and complex techniques because the noise variance $\left(\sigma_{1}^{2}\right)$ is re-calculated in every time of iterative LDPC decoder. Therefore, the noise variance $\left(\sigma_{1}^{2}\right)$ has always been changed and obtains more accurate value in each time of LDPC iteration. In contrast, conventional method is the worst in performance compared with others. Even though having exceptional capability, the complex technique requires a large of processing time for that result depending on the number of SNR iteration.

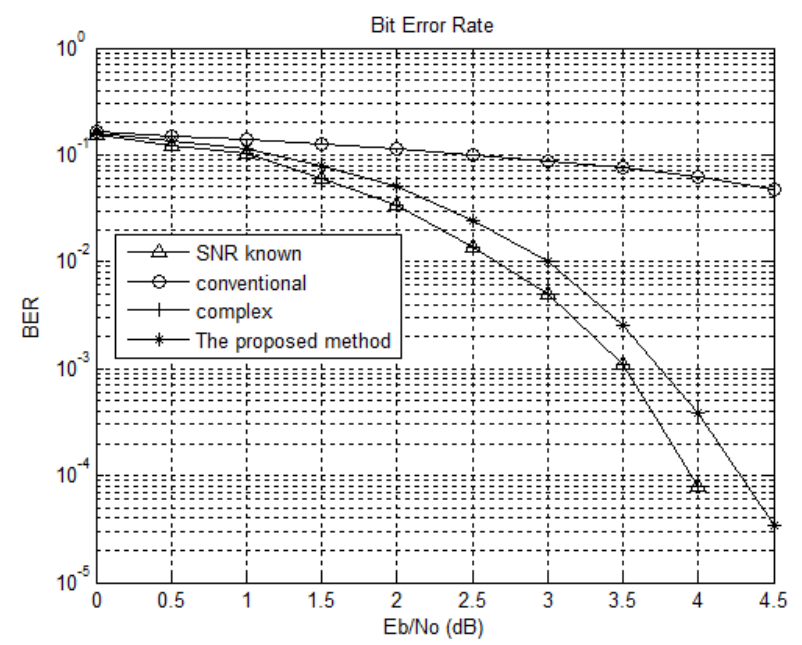

Figure 6. Bit-Error-Rate performance in the jiggling AWGN channel (the AR4JA (8192, 4096) code).

TABLE I. makes comparison of the single block information for SNR estimation of those three techniques. Generally, look up table is used to find the answer of exponential operation. Hence, three kind of operations are taken into our consideration namely addition, multiplication, and look up table.

Let length of single block information which pulsed RFI is erased is M. I is the number of iterative time of SNR estimation in the complex method. $\mathrm{k}$ represents the number of LDPC iteration. In the single block information, the conventional method requires the smallest operational numbers whereas the complex one demands a large amount of operation depending on number of I. The proposed approach has a little more computational complexity than the conventional technique depending on the number of LDPC iteration $(\mathrm{k})$. Moreover, the complex method must perform look up table process while the others are absent in this process.

For instance, in the multiplication operation, if the code length $(\mathrm{N})$ and pulsed RFI area are equal to 10240 and $4 \%$ of 
TABLE I. Comparisons of single block information for SNR estimation of three method.

\begin{tabular}{|c|c|c|c|}
\hline & $\begin{array}{c}\text { Conventional } \\
\text { method }\end{array}$ & $\begin{array}{c}\text { Complex } \\
\text { method }\end{array}$ & $\begin{array}{c}\text { Proposed } \\
\text { method }\end{array}$ \\
\hline Addition & $\mathrm{M}-1$ & $\mathrm{M}+(2+3 \mathrm{M}) \mathrm{I}$ & $(\mathrm{M}-1) \mathrm{k}$ \\
Multiplication & $\mathrm{M}+1$ & $3(1+\mathrm{I})+2 \mathrm{M}(1+2 \mathrm{I})$ & $(\mathrm{M}+1) \mathrm{k}$ \\
Look up Table & - & $4 \mathrm{MI}$ & - \\
\hline
\end{tabular}

code length respectively, eliminated pulsed RFI signal (M) is equal to $10240-(10240 \times 0.04) \approx 9830$. In the simulation, we set $\mathrm{k}$ and $\mathrm{I}$ by 50 and 100 respectively. According to TABLE I., the proposed approach succeed in decreasing the complexity in the multiplication operation by $87.56 \%$ compared with the complex method.

\section{CONCLUSION}

Joint iterative channel estimation and decoding has been proposed in this paper in order to mitigate the impact of pulsed RFI. The proposed approach presents joint iteration between channel evaluation and decoding technique so as to obtain more accurate the noise variance. The results show that the proposed technique has ability to attain less complexity computation than the complex method and enhance in the efficiency compared with the conventional method. Especially in the time varying SNR mismatch over AWGN channel. Therefore, the proposed approach is attractive to apply to various applications which pulsed RFI is involved with their requirements.

\section{ACKNOWLEDGMENT}

The authors would like to thank the full scholarship from Asia-Pacific Space Cooperation Organization (APSCO) and the China Scholarship Council (CSC), Scholarship No.2011GXZH34. We also grateful to thank the reviewers for their detailed reviews and constructive comments, which have helped to improve the quality of this paper.

\section{REFERENCES}

[1] (2011) Soft-Decision-Data Reshuffle to Mitigate Pulsed Radio Frequency Interference Impact on Low-Density-Parity-Check Code Performance[Online]. Available: http://ntrs.nasa.gov/

[2] David R. Pauluzzi and Norman C. Beaulieu, A Comparison of SNR Estimation Techniques for the AWGN Channel. IEEE TRANSACTIONS ON COMMUNICATIONS, vol.48, no.10, pp.1681-1691, Oct. 2000.

[3] Bin Li, Robert DiFazio, A Low Bias Algorithm to Estimate Negative SNRs in an AWGN Channel. IEEE COMMUNICATIONS LETTERS, vol.6, no.11, pp.469-471, Nov. 2002.

[4] Arun Ayyar, Michael Lentmaierl, K. Giridhar and Gerhard Fettweisl, Robust Initial LLRs for Iterative Decoders In Presence of Non-Gaussian Noise. IEEE International Symposium on Information Theory, 2009. p.904 - 908 .

[5] WILliAM E. RYAN and SHU LIN, Channel Codes Classical and Modern, 1st ed. Cambridge, England: Cambridge University Press, 2009.

[6] Peter F. de Bakker, Effects of Radio Frequency Interference on GNSS Receiver Output. Delft University of Technology, Netherlands.

[7] Christopher S. Ruf, Roger De Roo, Steve Gross, and Sidharth Misra, Radiometer Development of an Agile Digital Detector for RFI Detection and Mitigation on Spaceborne Radiometers. NASA Science Technology Conference, Maryland, USA, 2007.
[8] Christopher Ruf, Sidharth Misra, Steve Gross and Roger De Roo, Detection of RFI by its Amplitude Probability Distribution. Geoscience and Remote Sensing Symposium, 2006. IGARSS 2006. IEEE International Conference on, Denver, CO, USA, 2006. p.2289 - 2291.

[9] The Consultative Committee for Space Data System (CCSDS), LOW DENSITY PARITY CHECK CODES FOR USE IN NEAR-EARTH AND DEEP SPACE APPLICATIONS. Available: http://public.ccsds.org/default.aspx

[10] Mohammad Shafieipour, Heng-Siong Lim, Teong-Chee Chuah1, Decoding of Turbo Codes in Symmetric Alpha-Stable Noise. ISRN Signal Processing, Volume 2011 (2011), Jan. 2011.

[11] Ben Maad, H., Goupil, A.,Clavier, L., Gelle, G., Asymptotic performance of LDPC codes in impulsive non-Gaussian channel. Signal Processing Advances in Wireless Communications (SPAWC), 2010 IEEE Eleventh International Workshop on, Marrakech, Jun. 2010, p.1 - 5.

[12] Chuah, T.C., Robust iterative decoding of turbo codes in heavy-tailed noise. Communications, IEE Proceedings-(Volume:152, Issue: 1), pp. 29 - 38,Feb. 2005.

[13] Chuah, T.C., Decoding of low-density parity-check codes in nonGaussian channels. Communications, IEE Proceedings-(Volume:152 ,Issue: 6), pp. 1086 - 1097, Dec. 2005

[14] Chuah, T.C., Turbo equalisation in non-Gaussian impulsive noise. Vision, Image and Signal Processing, IEE Proceedings -(Volume:152 ,Issue: 1), pp. 52 - 60, Feb. 2005.

[15] Pan Xiao-fei, Hao Xie-Dong, A Joint Timing Recovery and SNR Estimation Algorithm for LDPC-Coded Systems. Wireless Communications, Networking and Mobile Computing, 2006. WiCOM 2006.International Conference on, Wuhan, Sept. 2006, p. 1 - 5.

[16] Seung Young Park, Yeun Gu Kim, Chung Gu Kang, Dae Eop Kang, Iterative receiver with joint detection and channel estimation for OFDM system with multiple receiver antennas in mobile radio channels. Global Telecommunications Conference, 2001. GLOBECOM '01. IEEE (Volume:5 ), pp. 3085 - 3089, San Antonio, TX, USA, 2001.

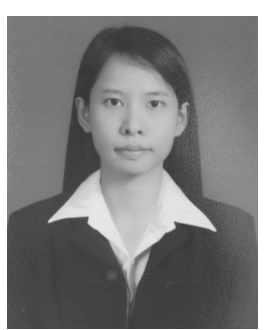

Patcharin Insom received the B.E. degree in Telecommunication Engineering (Second Class Honors), 2009 from King Mongkut's Institute of Technology Ladkrabang, Bangkok, Thailand. In 2013, she received the M.E degree in space application technology from Beihang University, Beijing, China. She now is Ph.D candidate at Chinese Academy of Science and interested research interested is remote sensing applications.

Rongke LIU received the B.S. and

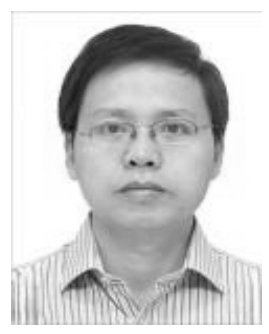
Ph.D. degrees in electronic and information engineering from Beihang University, Beijing, P.R. China, in 1996 and 2002, respectively. He is currently a professor of Electronic engineering department, Beihang University, China. His main areas of research interest are aerospace information transmission, telemetry and telecontrol and integrated circuit design. 


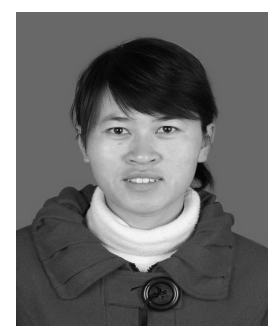

Ruifeng DUAN received the B.S. degree in electronic information science and technology from Inner Mongolia University, in 2008. She began to study in Beihang University as a Ph.D. candidate since 2009, in electronic and information engineering. She focus on detecting and acquisition for weak signal, and advanced coded modulation. She is interested in receiver technology in communication and telemetry.

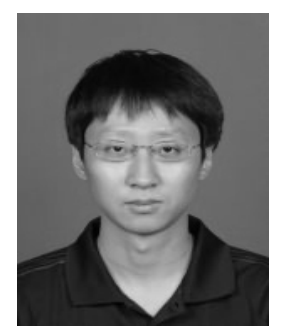

Yi HOU received the B.S.degree in electronic and information engineering from Beihang University, Beijing, P.R. China, in 2007. He is currently a Ph.D. student at the same university. Current research focuses on efficient channel coding and its implementation.

Pisit Boonsrimuang received the B.E. and M.E. degrees in telecommunication engineering from King Mongkuts Institute of Technology Ladkrabang (KMITL), Thailand and Dr.E. from Mie University, Japan, in 1997, 2000 and 2007 respectively. $\mathrm{He}$ is currently working as Vice President at King Mongkuts Institute of Technology Ladkrabang, Thailand. His research interests include transmission techniques for future multimedia wireless LAN systems. 